\title{
Los valores en la vida cotidiana
}

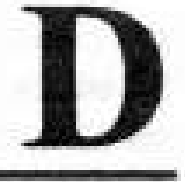

JOSE HOOVER VANEGAS GARCIA

Deeente Departamento de Ciencias Humanas

Universidad Autónoma de Manizales

Candidato a Magister en Filosofia con Enfasis en

Epistemologin escripción del problema

¿Como se entienden los valores en nuestra cultura fracturada por la falta de compromiso y lacerada por las intolerancias?; la forma de interpretar la valencia ${ }^{1}$ de las acciones que nos ha inculcado en la educación, a todo nivel, no ha funcionado, como lo demuestran los momentos de crisis por los que esté pasando Colombia en particular y el mundo en general. ¿Qué son, entonces los valores? ¿Qué significa educar en valores? Es necesario rescatar lo valores perdidos o significarlos de nuevo?.

Estos interrogantes nos exhortan a reflexionar sobre la labor que debe de desempefiar la ensettanma de la moral en nuestra época y especificamente la apreciación que los hombres desarrollan frente a las acciones como la matanza de $\mathrm{X}$ número de seres humanos en sólo una hora: ¿qué significa la muerte para las nuevas generaciones?. $Y$ por ende ¿qué valor tiene la vida en nuestra época?. Es el amor sinónimo de sexo, o por el contrario, es el amor la posibilidad racional que posee el hombre para tras- 
cender los límites de la corporalidad del otro?. ¿Qué sentido tienen en nuestro tiempo los actos honestos, solidarios, leales, responsables, entre otros?.

Este pequefio trabajo sólo pretende exponer unos criterios básicos para entender el problema de los valores e intentar rescatar la axiologia de las manos de la popularidad, puesto que todo lo que está de moda, como la moral, en estos tiempos y, por consiguiente, los valores, se creen tan bien entendidos que se pierde todo su sentido de comprensión, es decir, se vuelve trivial y, como tal, se manipula con sus falsos significados.

La base de estas especulaciones están escritas en el texto de Adela Cortina El Mundo de los Valores; los mismo que en Scheller en su obra Der Formalismus in der ETHIK und die materiale WERTETHIK. (El Formalismo en la Ética y los Valores Materiales), y otros textos auxiliares que enunciaremos en su debido momento

\section{¿Qué son los Valores?}

El hombre vive en un mundo en donde necesita de los otros y, ¿por qué no enunciarlo?, vive desde los otros. Somos nacidos para vivir en comunidad pero además tenemos la capacidad de conocer el mundo, divertimos con él, y hablar sobre él. Quizà la caracteristica más importante del hombre es haber desarrollado ese gran instrumento exosomático, el lenguaje articulado, El hombre puede describir el

\section{El hombre como corporalidad presente en el mundo fisico dis- pone los objetos de acuerdo con su aprecio, por esto, el cuerpo es el punto cero a partir del cual el mundo se ordena.}

mundo y determinarlo por medio de la matemática y la geometria o, por lo menos, hacerie creer a otros que asi lo hace.

Pero igualmente, con el lenguaje podemos juzgar sobre lo conocido, podemos lanzar al espacio infinito de los oidos sordos de nuestra época juicios evaluativos sobre cosas y acciones en el mundo que supuestamente son subjetivos: lo que es bueno y bello para mi, no tiene que serio para todo el mundo, se dice cotidianamente: acaso no es este uno de los grandes problemas de la valia que representa el hombre frente al mundo de los actos.

El lenguaje, nos dice Adela Cortina puede describir el mundo, por ejemplo el agua esta compuesta de dos átomos de hidrógeno y uno de oxigeno, enunciados con estas caracteristicas conforman el ámbito de las ciencias naturales, son descripciones de las cuales podemos verificar su verdad o falseded, no obstante: "Con el lenguaje prescriptivo, sin embargo, lo que intentamos es - como su nombre indica - prescribir la conducta; entendiendo ahora por "prescribir" dar orientaciones para que las personas actúen en consonancia"

El lenguaje prescriptivo, entonces, es normativo, por ejemplo: ser honesto es bueno, este enunciado se complica para concretar su verdad o falsedad, ya que no describe nada en el mundo sino que exhorta a una conducta determinada por medtio de una apreciación, sin embargo, su valor puede evaluarse como bueno o malo de acuerdo con el beneficio que su ejecución le pueda traer a la bumanidad. Este es el argumento que objetiva las acciones conductuales del hombre exteriorizadas por medio de un acto y significadas en una proposición prescriptiva.

En realidad, vivimos rodeados de valores, todo cuanto se nos da en el frente tiene un valor, la mesa que espera ser apoyada, la computadora que espera ser ordenada, el teléfono que espera ser vehiculo de la comunicación, absolutamente todo lo que está 
alrededor posee un valor, el color y la forma de la mesa son agradables a mis ojos y mi tacto, la computadora es asombrosa frente a mi ignorancia sobre ella, igualmente el teléfono, su caracteristica ergonómica satisface la forma de mi mano y la distancia entre misónganos fonadores $y$ auditivos. Vivimos rodeados de objetos valorados.

El mundo posee una dimensionalidad de acuerdo con la valencia que los objetos tienen para los individuos $y$ para ta sociedad en general. El hombre como corporalidad presente en el mundo fisico dispone los objetos de acuerdo con su aprecio, por esto, el cuerpo es el punto cero a partir del cual el mundo se ordena. Todos los objetos están situados en referencia a la co-preferencia de la intercorporalidad. El mundo, además de su estructura fisica constituye una representación valorativa para las personas.

Una casa, por ejemplo, posee una distribución de acuerdo con unos valores determinados por tradición: la sala es el lugar público, en donde se atienden las visitas, es el lugar más aparente del hogar; las habitaciones, por el contrario, son un lugar privado, por ello su disposición es más individual; sin embargo, los dormitorios no tienen la valia de privacidad que tiene el baño, este es un lugar individual, alli es en donde el hombre se automanifiesta sin ninguna pre- tensión, allise exterionzanjos mas intimos secretos del sí miscítidida. cocina se puede leer como elespacio de coparticipación comunicativa. En este lugar se tejen los comentarios 0 , los chismes, como se les llama comunmente.

Este ejempio lo podriamos aplicar a cualquier lugar cronotopológico, al edificio, al barrio, a la urbe, $y$ aun al pais. 0 Alrededor de este "yo" que valora se presentan igualmente seres humanos, otros que como yo son causa de la valoración, los cuales a su vez son objetos de $\mathrm{mi}$ apreciación, es decir, los hombres varlen en cuanto seres-cosas, presencias corporales, intermitencias en mí estimación. Pero no sólo valen como cuerpos, sino como trascendentalidades, como otro yo que ama, que sufre, que siente, o sea, como personas libres.

Como corporalidad, el otro se me presenta, en cuanto alteridad, como un extraño. En las últimas decadas el cuerpo ha cobrado un valor peculiar como significación intracorporal, desmembrada Primero, los usos postizos de pelucas, uñas, pestañas, entre otras. En la actualidad se nos imponen nuevos avances tecnológicos: transplantes de ojos, de órganos en general, prótesis que conducen a la perfección somatológica, en contra de las patologias.

Pero igualmente se imponen toda 
clase de somatoplástiscas, implantes de silicona con fines estéticos, protuberancias más eróticas, con el fin de suplir la idea de belleza que nos venden los medios de comunicación. El cuerpo ya no posee significación integral, pues aun en los rituales de coqueteo lo importante son una piemas provocativas, sin importar la integralidad de la persona.

Aunque este fenómeno no es nuevo, puesto que en la Biblia misma encontramos el surgimiento de Eva de una parte de Adán, en la actualidad se corre el peligro de reducir el ser humano a unas funciones especificas exteriorizadas en el cuerpo, lo cual significa una degradación por la inversión de los valores frente a la valia somatológica a ta que el hombre debe aspirar como ser humano.

El cuerpo humano también puede ser leído por fragmentos, la cara es el lugar publico, "La cana nos es más que una especie de tablero de instrumentos en el que desembocan todos los mecanismos del cuerpo: la digestión, la vista, la audición, la respiración, el pensamiento *. ${ }^{3} \mathrm{La}$ espalda se valora como el lugar de carga, piénsese en los morrales. Los órganos genitales, significan el lugar más privado,

Como persona, el otro me es dado a nivel moral, como libertad, como dignidad, el otro se me presenta como otro yo, que pien-
Sa, que siente, no como instrumento de mi voluntad, sino como un fin en si mismo, un extraño, un satélite de este yo que a su vez me alberga como el otro de él. Este yo es valorado por el otro como un sujeto de percepciones, como una intermitencia en donde elongamos nuestras brevedades. Somos cronotopológicamente, el uno distante del otro, pero sumergidos en valores pre-dados en una misma esfera, lo egológico.

En esta dimensión es en donde cobra sentido la ética y por extensión la moral, los cuerpos me son personas con carácter determinado, me son un proceso de ajustamiento de actos a realidades pre-dadas, tal como debo serlo yo para todos los otros. Porque el otro se me da en el frente $o$, en el recuerdoo, en la fantasía, porque este yo puede valorarlo desde sus actos, como otro que es justo, que es libre, que respeta, que es honesto... o, por el contrario: que es injusto, que es esclavo, que irrespeta, que es deshonesto.

Los valores morales determinan el grado de desarrollo humano que posee una colectividad o un individuo, si las apreciaciones de los hombres frente a los actos de los otros es positiva la sociedad es pacifica, si por el contrario la valencia frente a los actos es negativa, es una sociedad enferma, conflictiva. Pero no sólo lo dado al frente posee valor, iguaimente ...si las apreciaciones de los hombres frente a los actos de los otros es positiva, la sociedad es pacifica, si por el contrario, la valencia frente a los actos es negativa, es una sociedad enferma, conflictiva. 
este cuerpo que me acompana a todas partes, $\mathrm{mi}$ centinela y mi testigo, posee un valor, mis ojos valen porque ven, mis manos porque son herramientas para mi trabajo, $y$ aún más, mi cuerpo vale para el mundo de la misma forma que todo cuerpo que se me presenta alrededor vale para mi, asi sea solamente como un obstáculo que yo no puedo atravesar.

Sin embargo, no sólo lo tangible vale, lo no tangible también tiene un valor, por ejemplo, todos anhelamos la paz, no obstante, lo intangible de la significación de este término, la paz, textuaimente, no existe, pero ambicionamos que alguna vez exista, por lo menos que encontremos la mejor forma de convivir sin destruimos. Este concepto como tantos otros, por ejemplo: la justicia, la esperanza, entre otros, son valorados, son tenidos como ideales que buscan la máxima expresión de la racionalidad manifiesta en el comportamiento del hombre consigo mismo y por extensión con la otredad.

Ahora bien, no hay que confundir aprecio con precio; los valores inicialmente son apreciaciones que el hombre hace del mun-

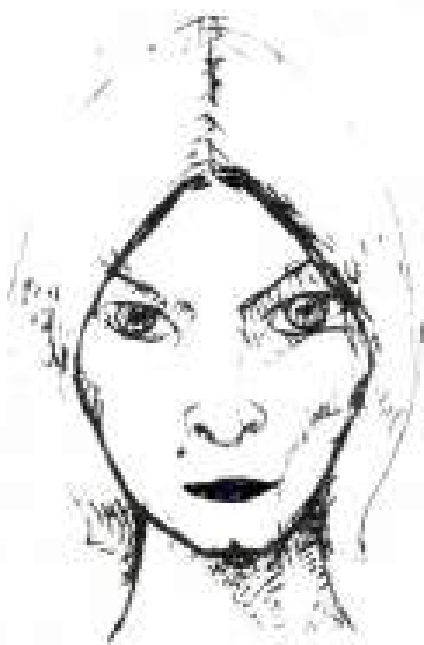

de asegurar un estilo de vida económica, y aún el desarrollo humano como conocimiento teórico se ha convertido en fuente económica; según esto el precio es cuantificable en un escala numérica, el aprecio es producto de la valia incuantificable que cobran determinadas cosas: personas, actos o conocimientos, para un individuo o para una sociedad. No hay que confundir precio con valor.

Esta confusión es uno de los productos de la degradación de un pueblo, en donde las personas, dejan de ser apreciadas como tales y empiezan a ser preciadas, esto es, a tener un precio económico. En la antigüedad algunos seres humanos carecian de la categoria juridica y moral de personas y eran tratados como esclavos, cran considerados como hombres-cosas, ésta es una de las peores degradaciones en la que pueden caer las civilizaciones, en estos tiempos, ya que ello se puede justificar en las épocas pristinas de acuerdo con el contexto racional que se vivia in illo tempore,

En la actualidad, esto supuestamente no se ve, no obstante, la manipulación sexual de do tanto real como ideal, el precio es una cuantificación económica que se elabora sobre un objeto tomando como base, entre otros, la utilidad; entre más útil sea un producto mayor será su coste.

La fuerza de trabajo tiene igualmente un valor per capita, en las sociedades consumistas, también tienen un valor económico las obras de arte que sin ser útiles, en sentido estricto, guardan un pedazo de historia y de belleza. En las últimas décadas ha surgido una nueva forma de capital, el conocimiento se ha convertido en un producto que se vende y que se compra, los saberes son en esta época una forma los medios de comunicación y el incremento de la prostitución corporal y auditiva de las últimas épocas, pero éste es tema para una investigación aparte.

Podemos afirmar que ei mundo posee un orden predado por las ciencias naturales, este orden es una imposición conceptual que se ha entretejido de la mano de la historia, los objetos en ei mundo existen yuxtapuestos cronotopológicamente dados para el hombre tanto a nivel de cosa como en valia.

Sin embargo los valores imponen un orden de una 
dimensión diferente a la valencia de los actos humanos en la medida en que ellos contribuyan al desarrollo normal del hombre, en general, es decir ${ }^{4} . .$. en descubrir creativamente el valor de la igualdad a pesar de las difenencias ". Si por el contrario los beneficios individuales persisten, se obstaculiza la evolución del hombre. Los valores son dispositivos que pretenden re-orientar las acciones humanas hacia el fin último de la racionalidad: la convivencia pacifica, la calidad humana.

Podemos concluir este apartado develando algunas caracteristicas de los valores. Cuando escuchamos hablar de antivalores podemos pensar que ello es un contrasentido puesto que un antivalor es en si mismo una forma del valor, no hay antivalores, los valores son bipolares, es decir, pueden ser negativos o positivos. El enunciado "carencia de valoress" es igualmente una contradicción, lo que sucede es que se valora negativamente, v.gr: no se respeta, sino que se es indiferente o se falta al respeto de la integralidad de los otros. Los valores, entonces, son valencias dinámicas que se dan en el mundo para determinar el comportamiento del hombre: "El valor se presenta como el resultado de una tensión entre el sujeto y el objeto y ofrece una cara objetiva y otra subjetiva. Ambos elementos no son. simples ni estables, sino complejos y cambiantes" 5

\section{Clasificación de los Valores}

Los juicios evaluativos traducidos en valores pue-

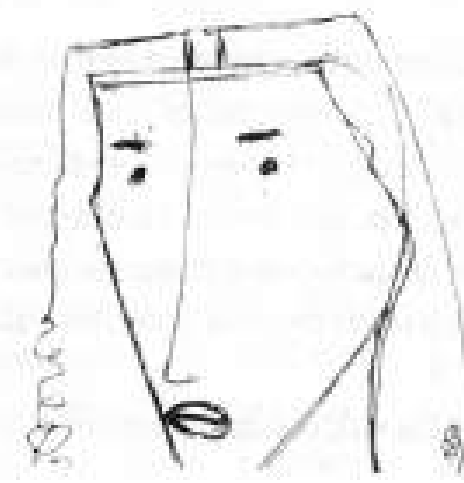

den ser de múltiples formas. En el siglo XIX Max Scheler filósofo alemán intentó una clasificación de ellos, incluia valores sensibles, vitales, religiosos y culturales. Más tarde el filósofo español Ortega y Gasset intento igualmente hacer una taxonomia de los valores. Sin embargo, en este trabajo adoptaremos la división que hace Adela Cortina. por considerarla más asequible, y aplicable a nuestras propias necesidades:

Sensibles (Placer/ Dolor: Alegria/ Pena).

Útiles (Capacidad/ Incapacidad; Eficacial Ineficacia).

- Vitales (Salud Enfermedad: Fortaleza/ Debilidad)

- Estéticos (Bello/ Feo: Elegante/ Inelegante: Armonioso/ Caótico)

Intelectuales: Verdad/Falsedad; Conocimiento/ Error.

Morales: Justicia/ Injusticia; Libertad/ Esclavitud; Igualdad/ Desigualdad; Honestidad/ Deshonestidad; Solidaridad Insolidaridad.

Religiosos: Sagrado/ Profano. ${ }^{6}$

Esta clasificación de los valores no implica que eilos son los únicos que se encuentran en el mundo, morada del hombre, en realidad hay muchos otros tales como la sublimidad y la insublimidad dentro de los valores estéticos; el respeto, la tolerancia y otros dentro de la valencia moral, sin embargo consideramos que tal clasificación recoge los elementos necesarios como para legitimar la clasificación de los valores.

En esta clasificación podemos ver varias caracteristicas, por ejemplo, hay valores que, en sentido estricto, dependen del sujeto o la sociedad valorante: el placer y la alegría son valores que dependen en mucha medida del sujeto inmerso en la vida cotidiana, no lo son de la misma forma los valores contrarios, los negativos: el dolor y la pena. Ellos dependen en gran medida de factores exteriores a los cuales está expuesto el hombre, tales como la falta corporal y hablada de un ser querido. 
En cuanto a los valores de utilidad, en un porcentaje alto, dependen de la persona valorante, sin embargo, no podemos decir lo mismo de las valencias vitales, la salud y la enfermedad se determinan bivalentemente. En parte, el sujeto que no previene, pero también los factores ambientales y sociales los determinan.

En cuanto a lo estético, lo bello, lo elegante, lo armonioso, lo sublime o lo feo, lo no elegante, lo caótico y lo no sublime de acuerdo con la sistematicidad dada a los sentidos del mundo exterior, es la labor de la aisthesis, la cual conforma el contexto del arte en general que hemos creado para valorizar el mundo desde el gusto

El contexto estético está elaborado por valores, éstos son el resultado de la expresión del hombre frente a la re-construcción del mundo real-empirico, son los juicios evaluativos que el hombre crea para manifestarse frente a lo agradable o desagradable como tal, los valores estéticos son significaciones intrinsecas a los objetos, en realidad lo bello y lo feo no dependen de la voluntad humana.

Los valores intelectuales conforman el criterio de objetividad de la ciencia, y más exactamente de las ciencias naturales. Son juicios elaborados "para afirmar de lo que es, que es y de lo que no es, que no es", parafraseando un poco a Aristóteles, en realidad ellos no dependen del hombre si no de la contrastación de los enunciados de las teorias cientificas con los hechos en el mundo.

Sin embargo, estos términos los usamos para valorar otros hechos en el mundo que no poseen la categoria de ciencia v.gr: es verdad que la esperanza es la luz que ilumina a los hombres en el devenir de la vida, no obstante los valores intelectuales en estos juicios prescriptivos pierden objetividad y se convierten en proposiciones problemáticas por su dependencia del enunciante.

En cuanto a los valores morales, de los cuales ya

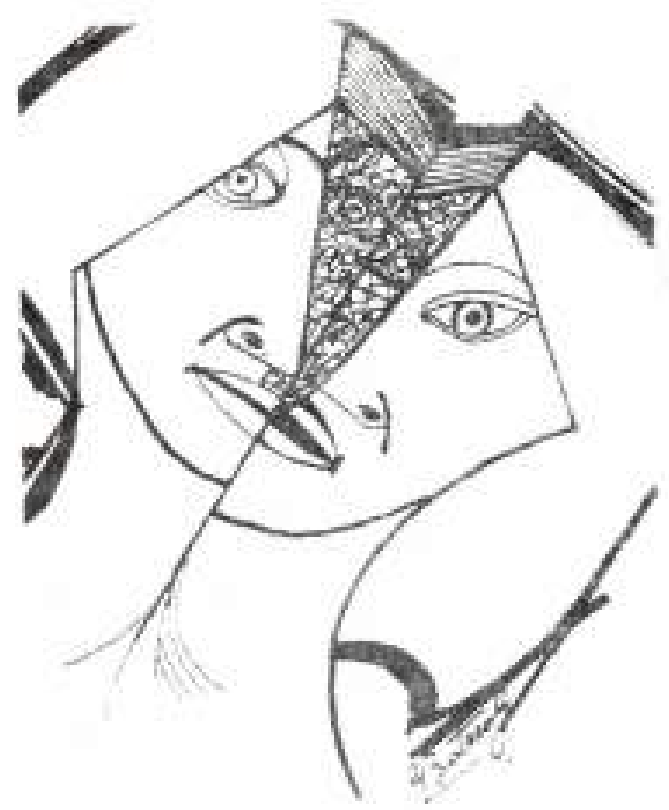

enunciamos su importancia, su legitimación depende de los individuos, es decir, ser solidario no es una facultad exterior al hombre, la solidaridad como todos los valores morales son en la medida en que la volición del hombre los actualice, y su objetividad depende del beneficio que pueda prestarle la cjecución positiva del valor al desarrollo humano o, en caso contrario, esto es, si se valora negativamente, insolidaridad; por ejemplo, se obstaculiza el desarollo humano.

En cuanto a los valores religiosos, lo sagrado y lo profano, ellos al igual que los morales son actualizados por los hombres.

Ahora bien, los valores poseen una jerarquia, de tal forma que frente a una situacion en la que se posean dos alternativas entre dos valores tanto positiva como negativa, hay que decidirse por el valor mas importante, por ejemplo: si alguien llega a mi casa buscando a un amigo, que en ese momento está de visita en mi residencia, para matarlo, yo debo preferir mentirle al posible homicida, antes que entregar a mi amigo a una muerte segura; en este momento yo prefiero mentir como la ejecución de un valor negativo puesto que esa misma acción esta salvando una vida y esta es superior que el de la mentira.

Hay otro elemento que no debemos dejar de men- 
cionar, los valores, como lo afirmábamos, son cualidades de los objetos, de los actos, o de los simbolos, y si tomamos en cuenta que en la actualidad estan surgiendo muevos objetos lo cual nos lleva ejecutar nuevas acciones y por lo tanto a elaborar nuevos simbolos; los valores toman nuevas significaciones en su dinámica de transposición.

Los nuevos programas para ordenadores modifican el comportamiento del hombre, el correo electronico, por ejemplo, Chatiar, como afirman las nuevas generaciones conduce a un nuevo ritual de coqueteo, a una nueva forma de comunicación, que en cierta medida está alimentando el anonimato de la presencia corporal, de la misma forma modifica el ejercicio de la educación éste analisis hay que hacerlo no solo frente al correo electrónico, sino tambien de acuerdo todos los avances cientificos, el sexo virtual, y con otras manifestaciones.

La aparición de nuevas forma de musica, el trans, la nuevas distribuciones de las ciudades, los condominios cerrados; el nuevo sentido del tiempo; los nuevos manejos de la libertad, la fragmentación social, la televisión en la educación, los video juegos; todos estos factores, entre otros nos dicen que los valores deben de ser re-significados en las nuevas creaciones exosomáticas. En otras palabras, los valores deben ser asimilados de acuerdo con los nuevos avances culturales que elaboran el dinamismo de la vida.

Esto significa que la división de los valores tal como la plantea Adela Cortina, no es estática, sino que ella tiene que estar en plena dialéctica en pleno devenir, si la finalidad es conseguir la mejor forma de vida en cuanto a la calidad humana.

\section{BIBL.JOGRAFIA}

ANGUREN, José Luis. Etica Barceiona: Altaya. 1994

- CORTINA, Adela. Razón Comunicativa y Responsabi- hidad Solidaria Salamanca: Sigueme, 1985.

__tica sin Moral. Madrid: Tecnos, 1990.

_- El Mundo de los Valores Etica Minima y Educacion. Santafé de Bogota, Segunda Edición: El Búho LTDA. 1998

ECO Humberto y MARTINI, Carlos Maria En que

Cree los que no Creen, Un Dialogo Sobre la Evica en

el fin del Milenio. Colombia. pianeta. 1998.

ESPINOSA, Baruch. Ética Dernostrada Segin ed Orden Geometrico. Barcelona Orbis. S.A. 1984

FRONDIZI, Risieri Pensamiento Axiologico, Colombia Universidad del Valle 1994

FROMM, Erich. EI Miedo a la Libertad

GUISAN, Esperanza. Razón y Pasión en Ética. Segunda Edición. Barcelons. Antropos 1990

HABERMAS, Jürgen. Escritos Sobre Moralidad y Eticidad. Barcelona: Paidós, 1991

HOTTOIS, Gilbert. El Paradigma Bioetico Barcelona: Antropos 1992

MITCHAN, Carl Qué es la Filosotia de ia Tecnologia? Madrid Antropos, 1989

- RAFHAEL D D Filosofia Moral Mexico: Fondo de Cultura Económico, 1986

- RAWLS, John. Teoria de la Justicia. Mexico: segunda edicion: FCE 1995

SANCHEZ Vazquez, Adolfo. Etica Meaco Grijalbo, 1969

- SAVATER, Femando. Ética como Amor Propio Barcelona: Grijalbo 1995.

- SINGER, Peter Pensar la Vida y la Muerte. Barcelona Paidós 199?

- SUZUKI, David y Knudtson, Peter Gentitica. Madnid Teenos. 1991

- VANEGAS, Jose Hoover El Deber en la Ética kantiana Universidad de Caldas. 1996

' Vaiencia y valia son terminos que Scheller usa para referirse a la labor de los valores.

${ }^{2}$ CORTINA, Adela. El Mundo de los Valores. Etica Minima y Educación. Santafe de Bogota: segunda edición. Editonal BihoLTDA 1998 . p. 20.

'KUNDERA, Milan La Insoportable Levedad del Ser Barcelona. RBA Editores. 1993 p: 44

Tbis p 70

'FRODIZI, risieri. Pensamiento Axiológico. Universidad del Valle : Biblioteca Americana 1994, p: 47

"Ibis 45. 